\title{
Antecedent Priming at Trace Positions in Children's Sentence Processing
}

\author{
Leah Roberts - Theodore Marinis • Claudia Felser • \\ Harald Clahsen
}

Published online: 9 December 2006

(C) Springer Science+Business Media, LLC 2006

\begin{abstract}
The present study examines whether children reactivate a moved constituent at its gap position and how children's more limited working memory span affects the way they process filler-gap dependencies. 46 5-7 year-old children and 54 adult controls participated in a cross-modal picture priming experiment and underwent a standardized working memory test. The results revealed a statistically significant interaction between the participants' working memory span and antecedent reactivation: High-span children $(n=19)$ and high-span adults $(n=22)$ showed evidence of antecedent priming at the gap site, while for low-span children and adults, there was no such effect. The antecedent priming effect in the high-span participants indicates that in both children and adults, dislocated arguments access their antecedents at gap positions. The absence of an antecedent reactivation effect in the low-span participants could mean that these participants required more time to integrate the dislocated constituent and reactivated the filler later during the sentence.
\end{abstract}

Keywords Children's sentence processing - Cross-modal priming - Antecedent reactivation · Filler-gap dependencies

\section{Introduction}

There is a growing body of literature investigating children's sentence processing in real time using on-line techniques that are familiar from adult sentence processing studies, such as self-paced reading and listening (Booth, Perfetti, \& MacWhinney 1999; Traxler, 2002; Felser, Marinis \& Clahsen, 2003), cross-modal priming (McKee, Nicol, \& McDaniel 1993; Love \& Swinney, 2007), eye-tracking (Trueswell, Sekerina, Hill, \& Logrip 1999; Sekerina, Stromswold, \& Hestvik, 2004), and event-related brain

L. Roberts · T. Marinis · C. Felser · H. Clahsen ( $ه)$

Department of Linguistics, University of Essex, Colchester CO4 3SQ, UK

e-mail: harald@essex.ac.uk 
potentials (Friederici \& Hahne, 2001). What emerges from these studies is that children from the age of 4-6years employ essentially the same parsing mechanism as adults and that any child-adult differences observed in these experiments can be attributed to other factors such as children's more limited working memory capacity, their reduced lexicon, or slower speed of lexical retrieval relative to adults. Consider, for example, the results from a self-paced listening study (Felser et al., 2003), investigating 6-7 year-old children's (and adults') processing of relative clause attachment ambiguities such as The doctor recognized the nurse of/with the pupil who was feeling very tired. The adults' preference to attach the relative clause to one of the potential NP hosts was affected by the lexical/thematic properties of the preposition linking the two potential antecedents. A similar trend was seen in a subgroup of children that had a relatively high working memory (WM) span, at least in an off-line (untimed) questionnaire. By contrast, children with low WM spans appeared to follow a local attachment strategy attaching the relative clause to the most recent host irrespective of the lexical/thematic properties of the preposition. These results suggest children's attachment preferences are not qualitatively different from those of adults and that child/adult differences may be attributable to differences in WM resources (see also Booth et al., 2000) for the demonstration of effects of memory span on children's processing of structurally complex sentences. A similar conclusion is drawn from the results of an eye-tracking study (Sekerina et al., 2004) with 5-7 year-old children investigating the processing of reflexive and non-reflexive pronouns. The children's eye movement data were found to be parallel to those of the adults, the only difference being that the children required more time than the adults to access sentence-external referents for pronouns, a finding that was attributed to children's reduced processing resources.

One area of sentence comprehension that has been intensively studied in adults is the processing of filler-gap dependencies in sentences such as [Which book] $]_{i}$ did Mary say [ John had read $\mathrm{t}_{i}$ ] in which the dislocated constituent which book is syntactically linked to its original direct object position. In generative-transformational theories of grammar (Chomsky, 1981, 1995), the syntactic link between these two positions is conceived of as a movement chain, with the original position hosting a syntactic gap ('trace'), i.e. a silent copy of the moved element. Antecedent reactivation effects in cross-modal priming experiments provide a major source of evidence indicating that syntactic gaps form part of the processing of sentence structure, by showing that at gap sites the moved constituent is mentally reactivated (see e.g. Nicol 1993; Love \& Swinney, 1996; Clahsen \& Featherston, 1999). Another important property of fillergap dependencies is that they present a challenge to the available processing resources insofar as the dislocated element must be held in WM until it can be reconstructed at its original structural position. Indeed, the computational cost incurred by temporarily storing a filler in WM has been found to increase with the distance between the filler and its associated gap (see, among others, King \& Just, 1991; Gibson 1998; Kluender \& Münte, 1998). Moreover, antecedent reactivation in filler-gap constructions has also been found to be affected by WM. In a cross-modal priming study examining longdistance scrambling constructions in Japanese, Nakano, Felser, and Clahsen (2002) obtained antecedent-priming effects at the gap position of a dislocated ('scrambled') direct object, but only for participants with a relatively high WM span. The low-span participants appeared to be unable to hold the filler in WM for long enough, i.e. across three intervening NPs, before encountering the gap site. 
Antecedent reactivation in children has so far only been examined by Love and Swinney (1997, in press). Building on this work, we have investigated children's processing of wh-dependencies using the cross-modal picture priming technique. We specifically ask whether children reactivate the filler at the gap site and how antecedent priming is affected by the children's WM span.

\section{Previous research on antecedent priming at gap positions}

Several studies using the cross-modal priming technique have found that adult listeners mentally reactivate a dislocated constituent at structural positions where Chomskian syntactic theory would posit a trace of that constituent. For example, Love \& Swinney (1996) studied English sentences containing object-relative clauses, such as Jimmy used the new pen that his mother-in-law recently \#1 purchased \#2, in which the object (= the new pen) is dislocated or moved to the left of the verb (= purchased) that subcategorizes the object. Love and Swinney (1996) found that lexical decision times at the position indicated by \#2 were significantly shorter for visual targets that were semantically related to the object of the embedded verb than for unrelated ones, whereas at the control position (= \#1) preceding the verb purchased, there was no such difference. These findings are consistent with the trace reactivation hypothesis, according to which the parser recovers or reactivates the grammatical and semantic features of the dislocated constituent at a potential gap site by creating a silent syntactic copy of the antecedent. Alternatively, Love and Swinney's results could also be explained in terms of direct association, according to which as soon as the subcategorizer is processed, a displaced argument will be linked to it directly (Pickering, 1993; Traxler \& Pickering, 1996). According to this view, reactivation effects for dislocated objects (e.g. pen in the sentence above) are the result of lexically processing the subcategorization frame of a transitive verb such as purchase and do not require the postulation of movement traces or syntactic gaps. One way of resolving this issue comes from studies investigating antecedent priming in head-final languages such as Japanese and German. Nakano et al. (2002) found that in the Japanese long-distance scrambling constructions in which a direct object is scrambled out from inside an embedded clause to the sentence-initial position, the dislocated object is reactivated at its gap site (see also Clahsen \& Featherston, 1999 for similar effects in German scrambling constructions). While these findings are compatible with the notion of trace reactivation, they are difficult to explain in terms of direct association, because (due to the head-final structure of Japanese and German) the antecedent-priming effect occurred before the subcategorizing verb has been encountered.

Antecedent priming in (4-6year-old) children has been studied by Love and Swinney $(1997,2007)$. They adopted the cross-modal picture priming (CMPP) task from McKee et al. (1993) to investigate object-relative clauses such as (1):

(1) [The zebra $]_{i}$ that the \#1 hippo had kissed $t_{i} \# 2$ on the nose ran far away.

Participants were given pictures at the two positions indicated in (1), (a) a picture of the dislocated direct object NP (e.g. zebra in (1), the 'related target'), (b) a picture of an unrelated animal (e.g. a camel for (1)), (c) a picture of an inedible object (e.g. a stone, the filler target). On the appearance of one of the targets, participants were required to make a decision as to whether or not the item in the picture was edible. The results obtained for children were similar to those of an adult control group. An antecedent-priming effect was found at the position indicated by \#2 in (1) in that the 
participants' decision times for related targets (e.g. for zebra in (1)) were significantly shorter than for unrelated ones, whereas at the control position $(=\# 1)$ there was no such difference. While this finding provides prima facie evidence for antecedent reactivation of dislocated constituents in children, it does not distinguish between trace reactivation and direct association, since both accounts predict an antecedent-priming effect at position \#2, but for different reasons. Clearly, more research is needed to determine whether children reactivate antecedents at gap sites.

\section{METHOD}

The aim of the present study is to investigate whether children show antecedent reactivation effects at gap sites and how such effects are affected by working memory. Participants were 46 children (mean age: 6.33, range: 5.9-7.0) and 54 adult controls (mean age: 22.8, range: 19-42) who had normal hearing and normal or corrected-tonormal vision. Parental consent was obtained prior to the children's participation in the study.

It is true that Love and Swinney (2007) have already shown antecedent priming effects for 4-6 year old children. However, given the relatively simple materials used by Love and Swinney (2007), it was not possible to decide whether this effect was due to trace reactivation or direct association of the dislocated antecedent with the subcategorizing verb. The specific purpose of the present study is to examine whether children show trace reactivation effects, i.e. antecedent priming at gap sites that are not adjacent to the subcategorizing verb. Consequently, the experimental sentences (see below) had to be more complex than those used by Love and Swinney (2007) involving three-place rather than of two-place predicates. We tested 5-7 year olds to be reasonably sure that they could handle the experimental materials.

The design of the experiment was modelled after Love and Swinney (2007). In addition to the main CMPP experiment, a picture-classification task and an auditory questionnaire were performed to validate the visual and language materials used in the main experiment. Moreover, to assess effects of WM, all participants undertook a standardized memory span test.

\section{Materials}

We tested sentences such as (2) below, i.e. object-relative clauses with a dislocated indirect object. As pointed out by Nicol (1993) in a cross-modal priming study with adult native speakers of English, the advantage of this construction is that the hypothesized trace position does not appear immediately adjacent to the subcategorizing verb, which should make it possible to dissociate trace reactivation from direct association effects. Specifically, only the trace reactivation account predicts a priming effect for the dislocated indirect object at the gap site, i.e. at the position indicated by $t_{i}$ in (2).

(2) John saw [the peacock $]_{i}$ to which the small penguin gave the nice birthday present $t_{i}$ in the garden last weekend.

Twenty experimental sentences were constructed for the CMPP task and ten for an additional auditory questionnaire (see Appendices A and B). To prevent subjects from developing any strategies in the CMPP task, 60 filler sentences were constructed 
matched in length to the experimental sentences. Of these, 48 included a variety of different constructions, and 12 were of the same type as the experimental sentences, but the visual targets were presented at different positions from those of the experimental sentences.

The visual targets for the CMPP experiment were (equal numbers of) pictures of animals or inanimate objects, all (except one) taken from Snodgrass and Vanderwart's (1980) set of pictures which are normed for children between the ages of 5 and 15 years. In addition, the nouns depicting the pictures for the identical and unrelated conditions were matched for syllable length and lemma frequency (Francis \& Kucera, 1982); see Appendix A for the picture labels shown in italicized capitals and how they were combined with the experimental sentences. For each experimental sentence, there were two visual targets, a 'related target', i.e. a picture of the indirect object noun (e.g. a picture of a peacock for (2) above), and an 'unrelated target', e.g. a picture of a carrot for (2). Visual targets were shown at two positions, at the trace position, i.e. at the offset of the final word of the direct object NP, e.g. after present in (2), and at a control position $500 \mathrm{~ms}$ earlier. Each experimental sentence was presented identically to four groups of subjects: the first one saw the related target at the trace position, the second group at the control position; the third group saw the unrelated target at the trace position, and the fourth group at the control position. Counterbalanced presentation lists were created for each subject group, so that there were equal numbers of related and unrelated targets in each list (see Appendix A for illustration). All visual materials were scanned, all auditory materials were read by a female native speaker of English and pre-recorded on a digital tape recorder.

\section{Procedure}

The experiments were administered individually in dedicated rooms in the children's schools or (with the adults) at the university. The CMPP experiment was administered in two sessions separated by at least 1 day with the children, and in one session with the adults. Each session started with six familiarization sentences, which were different for the two sessions. The same 20 experimental sentences and picture targets were used in each session, but - to avoid repetition effects - they were distributed over different conditions. If, for example, in the first session a picture target was presented at the control position, in the second session it was presented at the gap position, and vice versa. Moreover, if for a given sentence participants saw a related target picture in the first session, they saw an unrelated one for the same sentence in the second session. Prior to the CMPP experiment, the children were given a picture-classification task, and all participants completed an auditory questionnaire and a memory span test. Further details of the procedures are given below.

\section{Picture-classification task}

The pictures of the animals and inanimate objects used in the CMPP experiment were printed on cards, and the children were asked to sort these into two piles according to whether the picture on the card was alive or not alive. When participants were unsure of the aliveness of a given object, the experimenter trained them, until they all performed at ceiling on this task. 
Auditory questionnaire

Participants were instructed to listen carefully to a set of ten sentences and to answer a content question for each sentence which related to one of the three referents in the sentence; for each question, there were two possible answer options, as illustrated in (3). After listening to each sentence-question pair twice, the tape was stopped, and participants were asked to answer the content question.

(3) Paul waved at the bee to which the sweet ladybird gave a big bunch of flowers yesterday morning.

Question: Who did the ladybird give a big bunch of flowers to?

Answer options: Paul - the bee

Memory span tests

All participants undertook a memory span test. The adults underwent Daneman \& Carpenter's (1980) reading span test. Test materials consist of sets of two, three, four, five, and six sentences. The test sentences were displayed on cards one by one. Participants were asked to read the sentences aloud and to memorize the last word of each sentence at the same time. At the end of each set of sentences, participants were required to recall the final word of the sentences in each set in the order in which they had appeared. Reading span scores reflect the maximum number of sentences for which the participants could successfully recall the target words. The children underwent Gaulin \& Campbell's (1994) listening-span test, which is an adaptation of Daneman and Carpenter's task for children. The task involved listening to sets of one to six sentences, providing a truth-value judgment for each sentence, and then recalling the last word of each of the sentences at the end of each set.

\section{CMPP task}

Participants were seated in front of a 17" monitor, and instructed to listen carefully to the pre-recorded sentences over headphones. During the presentation of the sentences, pictures appeared on the computer screen, and the participants were required to decide whether the animal/object in the picture was alive or not alive, by pushing one of two buttons on a push-button box. Response times (RTs) were measured from the point at which the picture appeared on the screen to the participant's pressing of the response button. The presentation of the stimuli and the recording of RTs was controlled by the NESU software package (Baumann, Nagengast, \& Klaas, 1993). To ensure that the participants paid attention to the task, they were also asked to respond to 38 (yes-no) comprehension questions asking for one of the main characters. For example, for the experimental sentence Sue saw the hippo to which the tall giraffe gave the sweet tasty orange in the jungle yesterday afternoon, we asked Did Sue see the hippo? The comprehension questions were presented auditorily, randomly interspersed throughout the experiment following both experimental and filler sentences. To familiarize participants with the CMPP task, they were given two sets of practice tasks prior to the main experiment. The first set consisted of ten pictures of animals and inanimate objects presented on a computer screen for which they had to make an alive/not alive decision by pressing one of two buttons on a push-button box as 
quickly as possible. The second one required participants to make an alive/not alive decision whilst listening to sentences via headphones.

\section{Results}

Three accuracy measures indicate that the participants were able to perform the decision task properly and understood the kinds of sentences presented in the main experiment. The adults scored 96\% (SD: $2.5 \%$ ) correct on the comprehension questions presented during the CMPP experiment. One child scored 50\% and another $60 \%$ on the comprehension questions, i.e. accuracy scores 2 standard deviation (SDs) below the group's mean, indicating that they were not paying attention during the task; we therefore removed the data from these two children from any further analyses. Following the removal of these two children, the mean score for the child group rose to $86 \%$ (SD: $8.1 \%$ ). In the auditory questionnaire, the adults performed at ceiling, and the 44 children achieved an accuracy score of 87\% (SD: 1.04\%). Third, all participants scored highly on the accuracy of the alive/not alive decision required in the CMPP task; children $97 \%$ (SD: $3.7 \%$ ), adults $94 \%$ (SD: $2.6 \%$ ).

For analyzing the RT data, we followed common practice and included only those trials that were responded to correctly, removing trials for which the alive/not alive decision was incorrect. We also excluded extreme RTs of 2,000 ms or more for the adults (affecting $1.02 \%$ of the adult data) and 3,000 ms or more for the children (affecting $1.35 \%$ of the data points for the children). Finally, outliers, i.e. RTs that were more than two SD above or below the participant group's mean per condition were removed from the dataset.

Recall that in previous studies, the processing of filler-gap dependencies was found to be affected by the participants' WM. To examine whether this was also the case for the wh-dependencies tested here, we performed two preliminary ANOVAs, separately for the children's and the adult's RT data, with the within-subjects variables 'Position' (trace vs. control) and 'Target Type' (related vs. unrelated), and 'Memory Span' as a continuous variable. Both analyses revealed significant three-way interactions between these three variables (children: $F(1,42)=5.762 ; p=0.021$; adults: $F(1,52)=6.775 ; p=0.012$ ) indicating that memory span did indeed affect the processing of these constructions for both children and adults. To further investigate these interactions, we divided the children and the adults into two subgroups each according to their median memory span score, which was 2.5 for the children and 3 for the adults; this gave two subgroups of children, those with a relatively low listening $\operatorname{span}(\operatorname{span} \leqslant 2.5, n=25)$ and those with a high listening $\operatorname{span}(\operatorname{span}>2.5, n=19)$, and two subgroups of adults, a low-span group ( $\operatorname{span} \leqslant 3.0, n=32$ ) and a high-span group ( $\operatorname{span}>3.0, n=22$ ). Tables 1 and 2 present the mean response times to the visual targets for these participant groups.

To compare high-span children with high-span adults, we entered the RT data shown in Table 1 into an ANOVA with the between-subjects variable 'Group' (children vs. adults), and the within-subjects variables 'Position' (trace vs. control) and 'Target Type' (related vs. unrelated). This analysis revealed a 'Position' $\times$ 'Target Type' interaction $(F 1(1,39)=8.106, p=0.007 ; F 2(1,38)=8.596, p=0.006)$ and a main effect of 'Group' $(F 1(1,39)=83.824, p<0.001, F 2(1,38)=510.407$, $p<0.001)$. Planned comparisons following up on the two-way interaction revealed that RTs to identical targets at the trace position were faster than the RTs to unrelated 
Table 1 Mean RTs to visual targets for high-span participants

\begin{tabular}{lllll}
\hline & High-span children $(n=19)$ & \multicolumn{2}{l}{ high-span adults $(n=22)$} \\
\hline & Control position & Trace position & Control position & Trace position \\
Identical targets & 1,245 & 1,158 & 694 & 678 \\
Unrelated targets & 1,158 & 1,211 & 692 & 709 \\
\hline
\end{tabular}

Table 2 Mean RTs to visual targets for low-span participants

\begin{tabular}{lllll}
\hline & \multicolumn{2}{l}{ Low-span children $(n=25)$} & \multicolumn{2}{l}{ Low-span adults $(n=32)$} \\
\hline & Control position & Trace position & Control position & Trace position \\
Identical targets & 1,411 & 1,427 & 726 & 745 \\
Unrelated targets & 1,375 & 1,280 & 745 & 740 \\
\hline
\end{tabular}

targets in this position $(t 1(40)=1.991, p=0.053 ; t 2(19)=2.050, p=0.054)$ and faster than the RTs to the identical targets in the control position $(t 1(40)=1.707, p=0.094$; $t 2(19)=2.223, p=0.031)$. These results show that even though children had longer overall RTs than adults (hence the main effect of 'Group'), there was an antecedent-priming effect for these two participant groups (as revealed by the interaction of 'Position' and 'Target Type' which did not interact with 'Group'). Children and adults responded faster to related targets at the trace position than at the control position indicating reactivation of the indirect object at the gap position.

The same analysis was performed to compare low-span children to low-span adults (Table 2). As for the high-span groups, this analysis revealed a main effect of 'Group' $(F 1(1,55)=141.052, p<0.001 ; F 2(1,38)=175.129, p<0.001)$, an interaction of 'Position' and 'Target Type' which was significant for subjects and marginally significant for items $(F 1(1,55)=6.768, p=0.012 ; F 2(1,38)=3.171, p=0.083)$, and no significant three-way interaction $(F 1(1,55)=2.843, p=0.097 ; F 2(1,38)=1.447, p=$ 0.236). In contrast to the high-span groups, the ANOVA for the low-span participants also showed a main effect of 'Target type' $(F 1(1,55)=6.610, p=0.013 ; F 2(1,38)=$ $6.931, p=0.012)$, and an interaction between 'Position' and 'Group', which was significant for subjects $(F 1(1,55)=6.645, p=0.013)$ but not by items $F 2(1,38)=0.988$, n.s. $)$, as well as a 'Target Type' $\mathrm{x}$ 'Group' interaction $(F 1(1,55)=9.023, p=0.004$; $F 2(1,38)=9.832, p=0.003)$. Since the between-subjects variable 'Group' interacted with both 'Target Type' and 'Position', separate planned comparisons were performed on the subgroups of low span participants. For the low-span children, RTs to related targets at the trace position were significantly slower than to unrelated ones in this position $(t 1(24)=3.690 ; p=0.001 ; t 2(19)=3.300 ; p=0.004)$, the opposite of what was found for the high-span children. The RTs of the low-span adults were similar across conditions, in fact, none of the comparisons revealed any significant difference.

Considering, finally, the RTs at the control position, we note an unexpected contrast between the children and the adults. While the adults' RTs for both target types are similar to each other, the RTs of the children, in both span groups are longer for related than for unrelated targets. This contrast is illustrated in Table 3 in which the RTs to the two target types at the control position were collapsed for the two span groups. 
Table 3 Mean RTs to visual targets at control position

\begin{tabular}{lll}
\hline & Children $(n=44)$ & Adults $(n=54)$ \\
\hline Identical targets & 1,339 & 713 \\
Unrelated Targets & 1,281 & 723 \\
\hline
\end{tabular}

An ANOVA with the variables 'Target type' (related vs. unrelated) and 'Group' (adults versus children) revealed an interaction between these two variables which was significant for subjects $(F 1(1,96)=4.382 ; p=0.039 ; F 2(1,38)=2.736 ; p=0.106)$. This interaction reflects the fact that the children's (but not the adults') RTs for related targets were longer than for unrelated ones at the control position.

\section{Discussion}

The most interesting result of this experiment is the priming effect obtained at indirect object gaps in high-span children and adults. This finding receives a straightforward explanation from the trace-reactivation hypothesis according to which empty categories form part of the mental representations constructed during the processing of filler-gap dependencies. In our experimental sentences, the parser predicts an indirect object gap which reactivates the antecedent at the gap site and produces the observed priming effect. Note that the alternative direct association hypothesis, according to which a dislocated argument is directly linked to its subcategorizer, cannot explain the priming effect we found, as it occurred at a position that was not immediately adjacent to the subcategorizing verb. This finding is in line with the results of previous studies (on adults) in which antecedent-reactivation effects were obtained at trace positions that were clearly separated from the subcategorizing verb (Nicol 1993; Nakano et al., 2002; Clahsen \& Featherston, 1999). That antecedent-priming effects were not only found in adults but also in 5-7 year-old children (at least for the high-span subgroup) tallies with the results of Love and Swinney (in press) who obtained such effects with a CMPP task in 4 to 6 yearolds.

\section{Child-adult differences}

Child-adult differences were found at the control test point at which the children taken as one group took longer to respond to related than to unrelated targets (see Table 3). Love and Swinney (2007) also found longer RTs to related than to unrelated targets at the control position, even though this contrast only proved significant for their adult participants. We suggest that increased RTs for related targets can be explained as a lexical interference effect. Note that in the experimental sentences, the embedded subject always denoted an animal, e.g. a penguin in (2), and that in the condition 'related target at control position', a picture of another animal was shown, e.g. a peacock in (2), for which an alive/not alive decision was required. It is conceivable that the semantic features of these items interfered with each other causing a delayed response to the visual target. This was not the case for the unrelated targets which were pictures of inanimate objects, e.g. of a carrot, that were not semantically 
related to the kinds of nouns (i.e. animals) given as embedded subjects at the control position in the auditory stimulus.

\section{Effects of working-memory span}

We found effects of working memory capacity in both the children's and the adult's processing of filler-gap constructions in that only the high-span children and adults showed evidence of antecedent-priming at the gap position. For the low-span participants, however, there were no indications that the antecedent is reactivated at this position. Perhaps the low-span participants required more time to integrate the dislocated constituent and reactivated the filler at the word following the gap position or even at the end of the sentence, a possibility we cannot exclude since we did not test for reactivation effects at these positions. Interestingly, the lack of antecedent reactivation at the gap position seen in the low-span participants did not seem to compromise their ability to understand the experimental sentences, since they answered the comprehension questions that were asked after the auditory stimuli as accurately as the high-span participants. Hence it is possible that all that went wrong in the low span participants was that they 'forgot' the antecedent halfway through parsing the sentence, while nevertheless being able to provide a correct interpretation of the sentence. In any case, our finding that memory capacity is a relevant variable for discovering antecedent-priming effects is consistent with the results of earlier studies showing that the processing of complex sentences in general, and of fillergap constructions in particular, incurs a working memory cost in adults (Kluender \& Kutas, 1993; King \& Kutas, 1995; Kluender \& Münte, 1998; Miyamoto \& Takahashi, 2001; Nakano et al., 2002; Gibson \& Warren, 2004) and that for children, memory capacity may be a predictor of effective language processing (e.g., Booth, Perfetti, \& MacWhinney, 1999; Gathercole \& Baddeley, 1990, Gaulin \& Campbell, 1994; Booth et al., 2000).

Moreover, the two WM-span groups of children exhibited an interesting difference in their RT patterns. The low-span children had longer RTs to related than to unrelated targets at both test points, whereas the high-span children showed the reverse effect at the gap position. We explained the increased RTs at the control position as an interference effect, due to the similarity of the semantic features of the embedded subject noun in the auditory stimulus (e.g. penguin) and those of the noun in the visual target (e.g. peacock). With respect to the pattern found in the low-span children, it is conceivable that the embedded subject noun maintained some residual level of activation throughout the sentence which could have interfered with a semantically related visual target noun, not only at the control position but also further downstream in the sentence, and in this way yielded an interference effect at both test points. An interference effect was also found for the high-span children, but only at the control position. At the gap position, however, they showed the opposite pattern, i.e. a priming effect for related targets. Thus, for the high span children, the decisionmaking process on related targets is indeed facilitated, but only at the gap position, i.e. at a point during the processing of the auditory stimulus at which according to the trace reactivation hypothesis the parser reconstitutes the grammatical and semantic features of the antecedent. 


\section{Conclusion}

Our findings indicate that indirect object gaps access their antecedents during on-line sentence processing and that antecedent-priming effects are affected by working memory. These results were parallel for children and adults, as revealed by the priming effect obtained for high-span children and adults, but not for low-span children and adults. We also found differences between children and adults, i.e. longer response latencies to related targets than to unrelated ones in children (but not in adults), which were suggested to result from lexical interference rather than from any specific property of the children's parser. While these results and the interpretation given are compatible with a continuity view of language processing, according to which the parser is the same for children and adults and any performance differences between children and adults result from other factors (Crain \& Wexler, 1999), it would be interesting to see whether this is also the case for children at a younger age, e.g. for 2-3 year-olds. After all, it is possible that the parser undergoes a process of maturation and a period of qualitative change at early stages of language development, or that children 'learn' certain parsing mechanisms from experience. The empirical evaluation of these possibilities, however, requires experimental techniques that are suitable for studying on-line sentence processing in children at this age, and it is currently not clear what these techniques might be.

Acknowledgments The research in this paper was supported by the Leverhulme Trust (Grant No. F/00 213B to H. Clahsen, C. Felser, and R. Hawkins). We thank Ritta Husted, Michaela Wenzlaff, and the children at Kedall Primary School and St Thomas More's Catholic Primary School, Colchester, UK, for their participation, and the teachers and parents for their support. Leah Roberts is now working at the Max-Planck-Institute for Psycholinguistics, Nijmegen, and Theodore Marinis at the University of Reading.

\section{Appendix A: Sentences for the CMPP task $(n=20)$}

As illustrated for (1), each experimental sentence was used four times, yielding the following conditions: (a) related target presented at trace position (indicated by \#), (b) related target at control position, (c)unrelated target at trace position, (d) unrelated target at control position.

1. a. John saw the peacock to which the small penguin gave the nice birthday present \# in the garden last weekend.

PEACOCK

b. John saw the peacock to which the \# small penguin gave the nice birthday present in the garden last weekend.

PEACOCK

c. John saw the peacock to which the small penguin gave the nice birthday present \# in the garden last weekend.

CARROT

d. John saw the peacock to which the \# small penguin gave the nice birthday present in the garden last weekend.

CARROT

2. Sue called the spider to which the big ostrich showed the small pretty picture at his house yesterday evening.

BALLOON SPIDER

3. Jane loved the tiger to which the black beetle offered the sweet strawberry cake at the party last week.

TIGER

PAINTBRUSH

4. James phoned the giraffe to which the gray hippo told the scary ghost story in his room before bedtime.

VIOLIN

GIRAFFE 
5. Sue phoned the zebra to which the old rhino sent a short thankyou letter at his house yesterday morning. ZEBRA

HAMMER

6. George fed the panda to which the large leopard explained the difficult new game in the garden last Friday.

APPLE

PANDA

7. Fred chased the rabbit to which the brown eagle gave the small chocolate biscuit in the park last Monday. $R A B B I T$

NEEDLE

8. Bob loved the monkey to which the fat squirrel showed his excellent new trick in the playground last month. TOOTHBRUSH MONKEY

9. Ben liked the lobster to which the young camel offered the delicious melon at the beach last Saturday.

LOBSTER

BUTTON

10. Jack knew the donkey to which the nice tortoise told his most naughty secret by the river last week.

ONION

DONKEY

11. Jo knew the ostrich to which the black spider explained the difficult problem at school last Monday.

OSTRICH

$B A L L O O N$

12. John called the beetle to which the fat tiger showed his favorite photographs in the playground yesterday afternoon. PAINTBRUSH

BEETLE

13. Sue saw the hippo to which the tall giraffe gave the sweet tasty orange in the jungle yesterday afternoon.

HIPPO

VIOLIN

14. Jane knew the rhino to which the big zebra told a really funny joke at school yesterday morning.

$H A M M E R$

RHINO

15. James hit the leopard to which the old panda offered a very large ice-cream in the cinema after his lunch.

LEOPARD

APPLE

16. George liked the eagle to which the brown rabbit gave the biggest piece of cake at the party last Saturday.

NEEDLE

EAGLE

17. Fred chased the squirrel to which the nice monkey explained the game's difficult rules in the class last Wednesday. SQUIRREL

TOOTHBRUSH

18. Bob fed the camel to which the pink lobster showed his new computer game at his office on Monday morning.

BUTTON

$C A M E L$

19. Ben saw the tortoise to which the gray donkey gave the small expensive gift at the party last weekend.

TORTIOSE

ONION

20. Jack liked the penguin to which the bright peacock sent a nice Christmas present in the post last year.

CARROT

PENGUIN

\section{Appendix B: sentences for the auditory questionnaire}

1. Paul waved at the bee to which the sweet ladybird gave a big bunch of flowers yesterday morning.

2. Mary smiled at the penguin to which the small dolphin showed a big sea turtle last weekend.

3. Stuart phoned the swan to which the naughty goose told a very scary story last night.

4. Rose patted the sheep to which the hungry wolf sent an invitation for dinner yesterday evening.

5. William chased the fox to which the smart rabbit told a big lie at his birthday party.

6. Sandra called the duck to which the old rhino explained his fishing secret last month. 
7. Jeremy visited the squirrel to which the blue parrot gave a nice birthday present at his party.

8. Alice fed the kitten to which the huge elephant gave a ticket to the cinema yesterday evening.

9. Jonathan saw the monkey to which the fat hippo offered a delicious lunch last week.

10. Betty frowned at the ostrich to which the smart giraffe showed his favorite present after school.

\section{References}

Baumann, H., Nagengast, J., \& Klaas, G. (1993). Nijmegen: New experimental setup (NESU) Max Planck Institute for Psycholinguistics.

Booth, J., Perfetti, C., \& MacWhinney, B. (1999). Quick, automatic and general activation of orthographic and phonological representation in young readers. Developmental Psychology, 35, 3-19.

Booth, J., MacWhinney, B., \& Harasaki, Y. (2000). Developmental differences in visual and auditory processing of complex sentences. Child Development, 71(4), 981-1003.

Chomsky, N. (1981). Lectures on government and binding. Dordrecht: Foris.

Chomsky, N. (1995). The minimalist program. Cambridge, MA: MIT Press.

Clahsen, H., \& Featherston, S. (1999). Antecedent-priming at tracepositions: Evidence from German scrambling. Journal of Psycholinguistic Research, 28(4), 415-437.

Crain, S., \& Wexler, K. (1999). Methodology in the study of language acquisition: A modular approach. In W. Richie T. Bhatia (Eds.), Handbook of child language acquisition, (pp. 387-425). SanDiego: Academic Press.

Daneman, M., \& Carpenter, P. (1980). Individual differences in working memory and reading. Journal of Verbal Learning and Verbal Behavior, 19, 450-466.

Felser, C., Marinis, T., \& Clahsen, H. (2003). Children's processing of ambiguous sentence: a study of relative clause attachment. Language Acquisition, 11, 127-163.

Francis, N., \& Kucera, H. (1982). Frequency analysis of English usage: Lexicon and grammar. Boston: Houghton Mifflin.

Friederici, A., \& Hahne, A. (2001). Development patterns of brain activity. In J. Weissenborn \& B. Höhle (Eds.), Approaches to bootstrapping: Phonological, lexical, syntactic and neurophysiological aspects of early language acquisition, Vol.2, (pp. 231-246) Amsterdam, \& Philadelphia: John Benjamins.

Gathercole, S., \& Baddeley, A. (1989). Evaluation of the role of phonological STM in the development of vocabulary in children: A longitudinal study. Journal of Memory and Language, 28, 200-213.

Gathercole, S., \& Baddeley, A. (1990). Phonological memory deficits in language disordered children: Is there a causal connection? Journal of Memory and Language, 29, 336-360.

Gaulin, C., \& Campbell, T. (1994). Procedure for assessing verbal working memory in normal schoolage children: Some preliminary data. Perceptual and Motor Skills, 79, 55-64.

Gibson, E. (1998). Syntactic complexity: Locality of syntactic dependencies. Cognition, 68, 1-75.

Gibson, E., \& Warren, T. (2004). Reading-time evidence for intermediate linguistic structure in longdistance dependencies. Syntax, 7, 55-78.

King, J., \& Just, M. (1991). Individual differences in syntactic processing: The role of working memory. Journal of Memory and Language 30, 580-602.

King, J., \& Kutas, M. (1995). Who did what and when? Using word- and clause-level ERPs to monitor working memory usage in reading. Journal of Cognitive Neuroscience, 7, 376-395.

Kluender, R., \& Kutas, M. (1993). Bridging the gap: Evidence from ERPs on the processing of unbounded dependencies. Journal of Cognitive Neuroscience, 5, 196-214.

Kluender, R., \& Münte, T. (1998). Subject/Object asymmetries: ERPs to grammatical and ungrammatical wh-questions. Poster presented at the 11th Annual CUNY Conference on Human Sentence Processing, Rutgers University.

Love, T., \& Swinney, D. (1996). Coreference processing and levels of analysis in object-relative constructions: Demonstration of antecedent reactivation with the cross-modal priming paradigm Journal of Psycholinguistic Research, 20(1), 5-24. 
Love, T., \& Swinney, D. (1997). Real time processing of object relative constructions by pre-school children. Poster presented at the 10th Annual CUNY Conference on Human Language Processing, Santa Monica.

Love, T., \& Swinney, D. (2007) The processing of non-canonically ordered constituents in long distance dependencies by pre-school children: a real-time investigation. Journal of Psycholinguistic Research.

McKee, C., Nicol, J., \& McDaniel, D. (1993). Children's application of binding during sentence processing. Language and Cognitive Processes, 8, 265-290.

Miyamoto, E., \& Takahashi, S. (2001). Antecedent reactivation in the processing of scrambling in Japanese. MIT Working Papers in Linguistics, 43, 127-142.

Nakano, Y., Felser, C., \& Clahsen, H. (2002). Antecedent priming at trace positions in Japanese long-distance scrambling. Journal of Psycholinguistic Research, 31, 531-571.

Nicol, J. (1993). Reconsidering reactivation. In G. Altmann, \& R. Shillcock(Eds.), Cognitive models of speech processing: The second Sperlonga meeting (pp. 321-350). Hove: Erlbaum.

Pickering, M. (1993). Direct Association and sentence processing: A reply to Gibson, and Hickok. Language and Cognitive Processes, 8, 163-196.

Sekerina, I, Stromswold, K., \& Hestvik, A. (2004). How do adults and children process referentially ambiguous pronouns? Journal of Child Language, 31, 123-152.

Snodgrass, J., \& Vanderwart, M. (1980). A standardized set of 260 pictures: Norms for name agreement, image agreement, familiarity and visual complexity. Journal of Experimental Psychology: Human Learning, \& Memory, 6, 174-215.

Traxler, M. (2002). Plausibility and subcategorization preference inchildren's processing of temporarily ambiguous sentences: Evidence fromself-paced reading. The Quarterly Journal of Experimental Psychology, 55A(1), 75-96.

Traxler, M., \& Pickering, M. (1996). Plausibility and the processing of unbounded dependencies: An eye-tracking study. Journal of Memory and Language, 35, 454-475.

Trueswell, J., Sekerina, I., Hill, N., \& Logrip, M. (1999). Thekindergarden-path effect: Studying on-line sentence processing in young children. Cognition, 73, 89-134. 